\title{
Psoriasis: A Sequela of Streptococcal Infection Similar to Acute Rheumatic Fever
}

Herbert B Allen *, Brett Miller, John Durkin and Suresh G Joshi

Drexel University College of Medicine Philadelphia, USA

*Corresponding author: Herbert B Allen, 219 N. Broad St., 4th floor, Philadelphia, 19107, USA, Tel: 215-752-5550; Fax: 215-762-5570; E-mail: herbert.allen@drexelmed.edu

Received date: March 29, 2016; Accepted date: April 15, 2016; Published date: April 22, 2016

Copyright: (C) 2016 Allen HB, et al. This is an open-access article distributed under the terms of the Creative Commons Attribution License, which permits unrestricted use, distribution, and reproduction in any medium, provided the original author and source are credited.

\begin{abstract}
We propose that psoriasis is a sequela of streptococcal infection similar to acute rheumatic fever (ARF). This hypothesis arises from many different lines of evidence: a markedly elevated serum anti-streptococcal antibody is present in plaque psoriasis. However, cultures are routinely negative in psoriasis because the Group A Streptococcus pyogenes "hides" inside cells and inside biofilms and is thereby unculturable. The activation of either arm of the immune system is likely related to whether internalization or biofilm formation is predominant. With internalization, we postulate it is the adaptive immune system and with biofilm formation, the innate system. Lastly, prolonged treatment with anti-GAS antibiotics is effective in psoriasis as in ARF.
\end{abstract}

\section{Description}

Acute rheumatic fever (ARF) is considered a prototypical sequela of streptococcal pharyngitis [1]. It has clinical manifestations in the joints, heart, and skin as well as other organs. In the heart and, especially in the mitral valve, the consequences of this disease are very destructive and result from antigen-antibody reactions [2]. This disease, however, has become almost non-existent, save for occasional outbreaks [3]. This is due to the treatment of the preceding pharyngitis with antibiotics (particularly penicillin or penicillin derivatives); it is also due to the continued treatment of ARF with a daily dose of penicillin.

We propose psoriasis is also a sequela of streptococcal infection similar to ARF. It has similar clinical findings [4] and a similar response to penicillin [5]. There are many lines of evidence to support this hypothesis: the first is epidemiologic-if there is no group A Streptococcus pyogenes (GAS) in the environment, there is no psoriasis. This is true above certain latitude in northern Europe as well as certain Pacific islands including the largest, Australia. (The indigenous Aborigines had no psoriasis prior to the arrival of the English prisoners due to the lack of GAS in the environment.) The prevalence of GAS increased with decreasing latitude with a resulting increase in the prevalence of psoriasis [6]. This gradually decreased towards the equator.

The next line of evidence is the presence of GAS itself: in guttate psoriasis [7] (psoriasis with many small scaling lesions), patients have a positive throat culture or a positive anti-streptolysin O (ASO) titer. The addition of penicillin is useful in the treatment of this variety of psoriasis.

In contrast to guttate psoriasis, the presence of GAS in plaque psoriasis has been obscured by the fact that the organism cannot be cultured, nor is it accompanied by an elevated ASO titer. Two factors lead to this reality including internalization [8], (Figure 1) and formation of biofilms (Figure 2) [9]. The GAS thus "hides" in the tonsillar epithelial cells or in biofilms, only to reappear when the organisms leave the cells and recolonize [10]. With internalization, this may occur as long as a year after entry; and, with biofilms, it occurs only on the rare occasion when "exporter" cells leave [11]. These exporter cells can rapidly form a new biofilm (There is then a very narrow "window" for culturability) [12]. Consequently, these organisms truly create a chronic infection with little evidence of their presence.

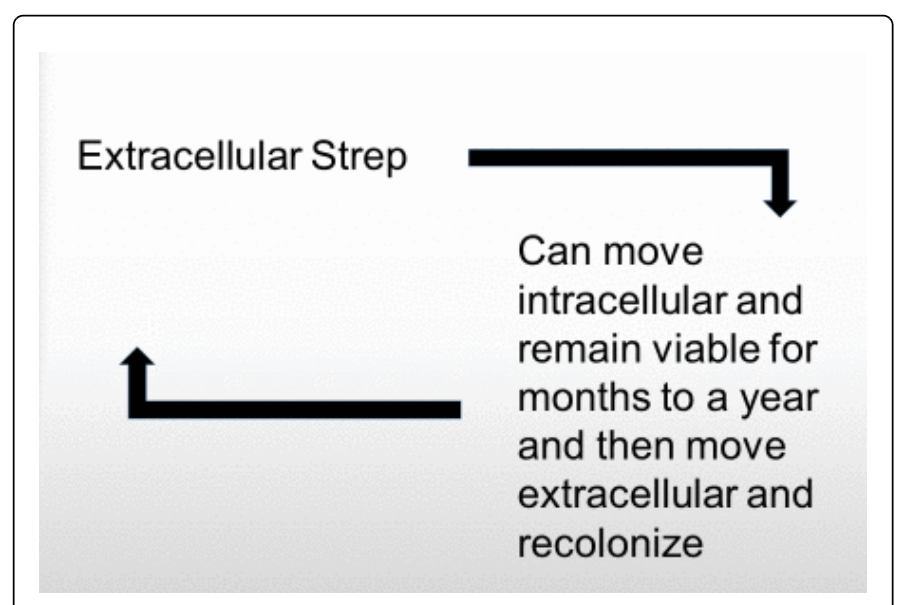

Figure 1: Schematic for Internalization of GAS.

\section{The Next Line of Evidence Comes from Treatment}

If plaque psoriasis is treated with continuing doses of penicillin, it resolves [13]. Penicillin likely kills the GAS being "externalized" before allowing them to react with the immune system. In like manner, it would kill the "exporter" cells as the leave the biofilm. Similar to ARF, the treatment needs to be continued for a long time. Surgical treatment has also been shown to ameliorate psoriasis [14]. This becomes a viable option especially when the psoriasis is accompanied by chronic tonsillitis or an elevated ASO titer. Similar to penicillin, clearing of the 
psoriasis may not occur for many months, as it takes that amount of time for the serum antibody to decay [15].

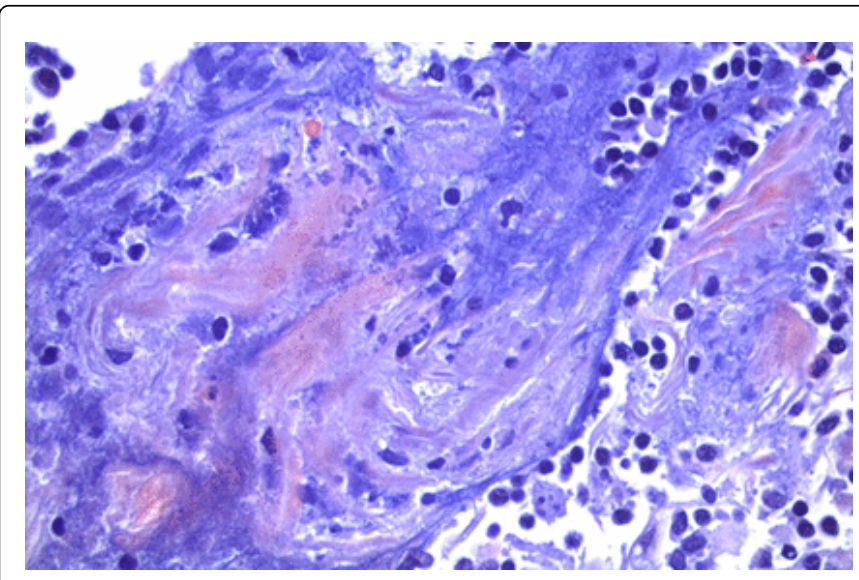

Figure 2: Tonsillar tissue from chronic tonsillitis in psoriasis. Congo red positive staining confirms the presence of amyloid which forms the infrastructure of biofilms. 40X.

The serum antibody versus GAS forms the next line of evidence. In plaque psoriasis, the anti-streptococcal IgG is markedly elevated compared to controls [16]. When the graph displaying this finding is examined, it is obvious that $60 \%$ of the patients had markedly elevated antibodies, "dragging" the entire group to the significant level seen. The remainder was in the "control" range. When one considers the behavior of the internalized GAS, a possible answer becomes apparent the bacterial cells externalize and the antibody has an anamnestic response leading to the markedly higher level found (Figure 3). The remainder of the patients in whom the GAS is in the biofilms, conceivably activate the innate immune system as in other diseases [17]. This would lead to the Toll-like receptor 2 that has been found in the dermal capillaries (Figure 4). There it can lead to the development of the psoriatic lesions by known pathways, most notably $\mathrm{MyD} 88 \rightarrow \mathrm{NF \kappa B} \rightarrow \mathrm{TNFa}[18]$.

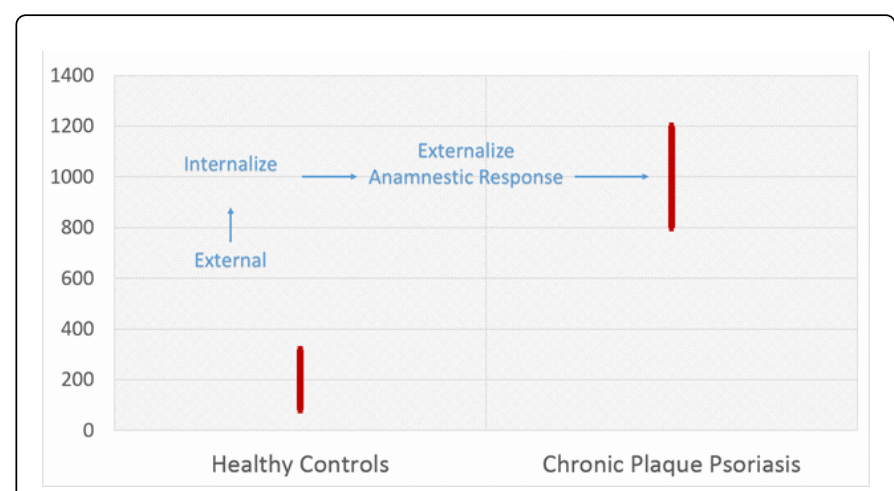

Figure 3: Combination of Figure 1 and adaptation of graph from reference 16; demonstrates how IgG could be markedly elevated in plaque psoriasis patients.

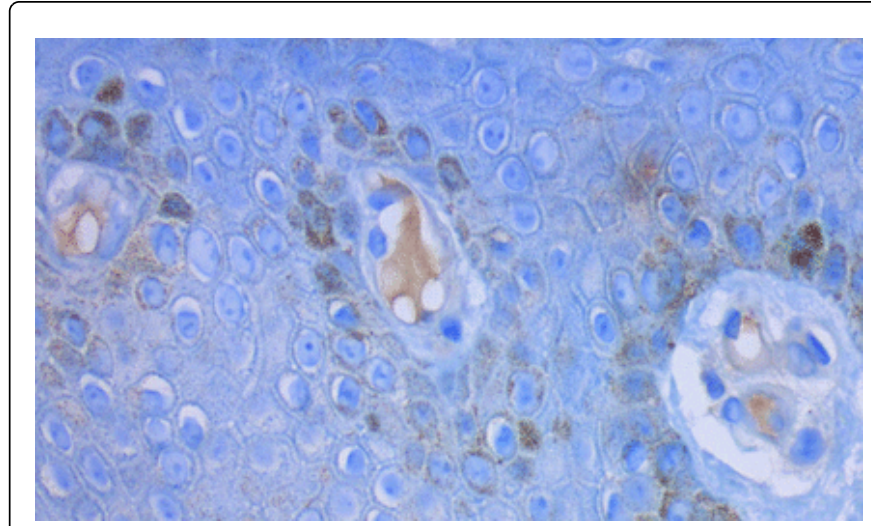

Figure 4: Biopsy of psoriatic plaque stained with CD 282 (TLR 2). Positive staining is noted in the lumens of the upper dermal capillaries and in the basal layer of the epidermis (control location in the epidermis). $40 \mathrm{X}$.

Compared to the innate immune system working primarily with $\mathrm{TNF} \alpha$, the adaptive immune system has many more weapons at its disposal: complement, alternate complement, killer $\mathrm{T}$ cells and many cytokines. Related to the elevated IgG, this would most likely lead to the development of the destructive psoriatic arthritis, chronic metabolic syndrome, renal disease, and uveitis noted in several patients. It would also be the ultimate comparator for psoriasis as related to ARF.

The antigen in the skin is yet to be determined as is the impact of the gene (one of the PSORS genes) [19]. It is likely to be via molecular mimicry as is the situation in ARF. Even more obscure are the antigens for the many varieties of psoriasis, such as pustular and erythrodermic.

The impact of the biologic medications on psoriasis has been dramatic. Those medications act very late in the cascade of events from the initial GAS infection to the psoriatic plaque. While very effective, these agents must be continued because they are not addressing the source of the inflammatory response. Perhaps, if the patient could tolerate it, the ideal treatment would be a biologic medication and penicillin plus/minus a biofilm disperser. The biologic predictably would cause remission and then could be discontinued after 10-12 months while the penicillin was continued. It was shown that the penicillin could even be discontinued after two years in many patients [13]. Presumably, these are the patients with internalization of the GAS; those with the biofilms would, in all probability, need the additional agents.

Thus, similar to ARF, psoriasis leads to involvement of other organs. Psoriasis is very destructive in the in the joints, while ARF is most destructive in the cardiac tissue. Moreover, psoriasis differs from ARF in that it will cause renal involvement similar to glomerulonephritis while ARF does not [20]. As previously postulated, this tissue destruction is very likely due to the adaptive immune system with involvement of an antibody. Consequently, with the involvement of the adaptive immune system, tissue destruction occurs much more extensively and with much greater alacrity leading to disabling disease $[17,21]$. 


\section{Summary}

We have shown how epidemiology, microbiology, physiology, immunology, clinical findings and treatment all play a role in the hypothesis that Streptococcus is most important in the etiology of psoriasis. We have also shown how it is not the presence of the organisms, or the process of internalization, or the biofilm production that actually leads to the disease. It is the immune system, in both its arms, innate and adaptive, which causes the disease. In nearly every regard then, it is similar to rheumatic fever, and we believe it should also be included as a sequela of streptococcal infection.

Genetics plays an important role, probably as the first (or second) hit in the double-hit phenomenon, and the streptococcus represents the environmental "hit". This can be seen when there is acute or chronic pharyngitis without the development of psoriasis because the patient lacks the gene.

Penicillin and azithromycin (both excellent anti-streptococcal drugs) have been shown to eradicate the psoriasis, and we have theorized why they must be continued for long intervals. Also, in many patients, the addition of a biofilm dispersing agent may be necessary.

All the foregoing should help in understanding of this cutaneous disease with systemic findings, and should firmly place it alongside rheumatic fever and glomerulonephritis as a sequela of streptococcus.

\section{References}

1. Chakravarty SD, Zabriskie JB, Gibofsky A (2014) Acute rheumatic fever and streptococci: the quintessential pathogenic trigger of autoimmunity. Clin Rheumatol 33: 893-901.

2. Cunningham MW (2012) Streptococcus and rheumatic fever. Curr Opin Rheumatol 24: 408-416.

3. Beaudoin A, Edison L, Introcaso CE, Goh L, Marrone J, et al. (2015) Acute rheumatic fever and rheumatic heart disease among children-American Samoa, 2011-2012. MMWR Morb Mortal Wkly Rep 64: 555-558.

4. Yeung H, Takeshita J, Mehta NN, Kimmel SE, Ogdie A, et al. (2013) Psoriasis severity and the prevalence of major medical comorbidity: population-based study. JAMA Dermatol 149: 1173-1179.

5. Allen HB, Kim J, Warner CW, Joshi S (2015) Penicillin: the new/old wonder drug. J Drug Metab 6: 37.

6. McFadden JP, Baker BS, Powles AV, Fry L (2009) Psoriasis and streptococci: the natural selection of psoriasis revisited. Br J Dermatol 160: 929-937.
7. Zhao G, Feng X, Na A, Yongqiang J, Cai Q, et al. (2005) Acute guttate psoriasis patients have positive streptococcus hemolyticus throat cultures and elevated antistreptococcal M6 protein titers. J Dermatol 32: 91-96.

8. Kaplan EL, Chhatwal GS, Rohde M (2006) Reduced ability of penicillin to eradicate ingested group A streptococci from epithelial cells: clinical and pathogenetic implications. Clin Infect Dis 43: 1398-1406.

9. Baldassarri L, Creti R, Recchia S, Imperi M, Facinelli B, et al. (2006) Therapeutic failures of antibiotics used to treat macrolide-susceptible streptococcus pyogenes infections may be due to biofilm formation. J Clin Microbiol 44: 2721-2727.

10. Wood DN, Chaussee MA, Chaussee MS, Buttaro BA (2005) Persistence of Streptococcus pyogenes in stationary-phase cultures. J Bacteriol 187: 3319-3328.

11. Allen HB, Vaze ND, Choi C, Hailu T, Tulbert BH, et al. (2014) The presence and impact of biofilm-producing staphylococci in atopic dermatitis. JAMA Dermatol 150: 260-265.

12. Allen H, Neidig L, Zhang J, Shaver C, Cusack C (2015) The etiology of psoriasis: its close association to streptococcus. J Am Acad Dermatol 72: AB254.

13. Saxena VN, Dogra J (2005) Long-term use of penicillin for the treatment of chronic plaque psoriasis. Eur J Dermatol 15: 359-362.

14. Rachakonda TD, Dhillon JS, Florek AG, Armstrong AW (2015) Effect of tonsillectomy on psoriasis: a systematic review. J Am Acad Dermatol 72: 261-275.

15. Johnson DR, Kurlan R, Leckman J, Kaplan E (2010) The human immune response to streptococcal extracellular antigens: clinical, diagnostic, and potential pathogenetic implications. Clin Infectious Dis 50: 481-490.

16. El-Rachkidy RG, Hales JM, Freestone PP, Young HS, Griffiths CE, et al. (2007) Increased blood levels of IgG reactive with secreted streptococcus pyogenes proteins in chronic plaque psoriasis. J Invest Dermatol 127: 1337-1342.

17. Allen HB, Shaver CM, Etzler CA, Joshi SG (2015) Autoimmune Diseases of the Innate and Adaptive Immune System including Atopic Dermatitis, Psoriasis, Chronic Arthritis, Lyme Disease, and Alzheimer's Disease. Immunochem Immunopathol 1:112.

18. Callahan JA, Hammer GE, Agelides A, Duong BH, Oshima S, et al. (2013) Cutting edge: ABIN-1 protects against psoriasis by restricting MyD88 signals in dendritic cells. J Immunol 191: 535-539.

19. Mahil SK, Capon F, Barker JN (2015) Genetics of psoriasis. Dermatol Clin 33: 1-11.

20. Wan J, Wang S, Haynes K, Denburg MR, Shin DB, et al. (2013) Risk of moderate to advanced kidney disease in patients with psoriasis: population based cohort study. BMJ 347: f5961

21. Allen HB, Morales D, Jones K, Joshi S (2016) Alzheimer's Disease: A Novel Hypothesis Integrating Spirochetes, Biofilm, and the Immune System. J Neuroinfect Dis 7:200. 